\title{
Aprendizaje Basado en Proyectos (ABP). Congreso on-line como metodología de investigación integral aplicada a estudiantes de postgrado en Bellas Artes
}

\section{Gloria Lapeña Gallego ${ }^{\mathrm{a}}$ y José Mayor Iborra ${ }^{\mathrm{b}}$}

aDepartamento de Dibujo. Facultad de Bellas Artes. Universidad de Granada. lapena@ugr.es bDepartamento de Bellas Artes. Facultad de Bellas Artes. Universidad de Murcia. jmayor@um.es

\begin{abstract}
Project-Based Learning (PBL) is a teaching method characterized by solving a real problem by using interdisciplinary knowledge. We propose a PBL in the subject "Methodology and Research in Fine Arts" of the Master of Production and Artistic Management (Faculty of Fine Arts, University of Murcia). The purpose is the development of an on-line congress. The aim is to provide postgraduate students with the tools and skills in order to disseminate their research results to the rest of the scientific community. Each student submits a video communication (researcher role), evaluates two anonymous proposals (referee role) and interacts in the discussion forums. We conclude that the satisfactory results of the initial prospective evaluation and the final self-evaluation are good indicators of the achievement of the aim proposed in this study.
\end{abstract}

Keywords: Project-Based Learning, teaching methodology, Fine Arts, postgraduate studies.

\footnotetext{
Resumen

El Aprendizaje Basado en Proyectos (ABP) es un método de enseñanza caracterizado por la resolución de un problema real utilizando conocimientos interdisciplinares. Proponemos el diseño de un ABP en la asignatura "Metodología e Investigación en Bellas Artes" del Máster Universitario en Producción y Gestión Artística impartido en la Facultad de Bellas Artes de la Universidad de Murcia. El objeto es el desarrollo de un congreso on-line y el objetivo dotar a los estudiantes de postgrado de las herramientas y habilidades para difundir sus resultados de investigación al resto de la comunidad científica. Cada estudiante presenta una video comunicación (rol de investigador), evalúa dos propuestas anónimas (rol de revisor) e interactúa a través de los foros de discusión. Concluimos que los resultados satisfactorios de la evaluación inicial prospectiva y de la autoevaluación final son un buen indicador de la consecución del objetivo planteado en este estudio.
}

Palabras clave: Aprendizaje Basado en Proyectos, metodología docente, Bellas Artes, estudios de postgrado. 


\section{Introducción}

El Aprendizaje Basado en Proyectos (ABP) o Project-based learning (PBL) es un método de enseñanza ampliamente utilizado en educación infantil, primaria y secundaria que tiene como finalidad solventar los principales errores del sistema educativo actual. Dos de estos errores son el aprendizaje memorístico de materias inconexas y la evaluación por parte del profesor mediante exámenes, sin tener en cuenta el desarrollo de todas las capacidades del estudiante. Su inclusión como metodología en la enseñanza no es reciente. Torrego Egido y Méndez Romero (2018) dedican un monográfico al ABP en la Revista Electrónica Interuniversiaria de Formación del Profesorado (REIFOP) con motivo del centenario de la publicación del ensayo de Kilpatrick, en el que describe y delimita este método por primera vez bajo la denominación "Project Method".

Jones, Rasmussen y Moffitt (1997) analizan ampliamente las características que debe cumplir la planificación y desarrollo de un ABP para que sea considerado como tal. En primer lugar, estará configurado por un conjunto de experiencias y de tareas de aprendizaje basadas en la resolución de un reto o problema. Todo el proceso girará en torno a la consecución de un proyecto, lo cual, además de la adquisición de conocimientos interdisciplinares, permitirá al alumnado poner en práctica modos de comportamiento social más próximos a la realidad que las metodologías tradicionales. En segundo lugar, va a ser el propio estudiante el principal implicado en el diseño y planificación del aprendizaje, en la toma de decisiones y en los procesos de investigación. Este aspecto tiene que ver con un trabajo autónomo que ocupa al estudiante la mayor parte del proceso. Por último, la culminación de un $\mathrm{ABP}$ será la entrega de uno o varios productos finales que se presentarán ante los compañeros, produciéndose interacción entre ellos.

El presente proyecto de innovación docente ha sido soportado por la Unidad de Innovación de la Universidad de Murcia (curso 2018/2019) y concedido bajo el título "Recreación de congreso on-line en el Aula Virtual para la puesta en práctica de la metodología de la investigación en Bellas Artes". Se vertebra en torno a la metodología del ABP, cuyo objeto de trabajo es un congreso on-line utilizando el Aula Virtual como plataforma de comunicación. Los destinatarios del ABP son los estudiantes de postgrado matriculados en la asignatura "Metodología e Investigación en Bellas Artes" del Máster Universitario en Producción y Gestión Artística impartido en la Facultad de Bellas Artes de la Universidad de Murcia.

\section{Objetivos}

El objetivo general del proyecto de innovación es dotar a los estudiantes de postgrado de las herramientas y habilidades para difundir sus resultados de investigación al resto de la comunidad científica a través de un hipotético congreso on-line.

Los objetivos específicos derivan de los distintos roles y capacidades que se generan a lo largo del desarrollo del proyecto o base de aprendizaje, destacando el rol de investigador y el de revisor por pares o iguales. 
Son objetivos específicos:

- Plantear una comunicación de investigación.

- Desarrollar una actitud crítica sobre la investigación de iguales.

- Elaborar una video comunicación oral.

- Participar de manera apropiada en foros de discusión científicos.

\section{Desarrollo de la innovación}

Para el cumplimiento de los objetivos específicos, los dos profesores integrantes de este proyecto actuamos de manera puntual como organizadores del congreso on-line, hacemos un seguimiento del trabajo personal a través de la tutoría electrónica, aportamos los recursos necesarios para la realización de las tareas y coordinamos los foros de discusión, delegando la mayor parte del proyecto en los propios estudiantes. Cada objetivo conlleva una tarea concreta que realiza el estudiante de manera individual y un sistema de evaluación en el que el profesor, e incluso el propio estudiante, asignan puntuaciones mediante rúbrica (Tabla 1).

Tabla 1. Tareas a desarrollar por el alumno en función de cada objetivo y su evaluación

\begin{tabular}{|c|c|c|}
\hline Objetivo & Tarea del estudiante & Evaluación \\
\hline $\begin{array}{l}\text { Plantear una } \\
\text { comunicación de } \\
\text { investigación. }\end{array}$ & $\begin{array}{l}\text { Acota tema de investigación, } \\
\text { selecciona palabras clave, realiza } \\
\text { búsqueda bibliográfica y sintetiza } \\
\text { en un resumen la propuesta. }\end{array}$ & $\begin{array}{l}\text { Evaluación del resumen } \\
\text { mediante rúbrica } \\
\text { (profesorado). } \\
\text { Evaluación mediante rúbrica } \\
\text { del resumen por pares } \\
\text { (estudiante). }\end{array}$ \\
\hline $\begin{array}{l}\text { Desarrollar una } \\
\text { actitud crítica sobre la } \\
\text { investigación de } \\
\text { iguales. }\end{array}$ & $\begin{array}{l}\text { Evalúa el trabajo elaborado por dos } \\
\text { compañeros anónimos. }\end{array}$ & $\begin{array}{l}\text { Evaluación mediante rúbrica } \\
\text { de la evaluación por pares } \\
\text { (profesorado). }\end{array}$ \\
\hline $\begin{array}{l}\text { Elaborar una video } \\
\text { comunicación oral. }\end{array}$ & $\begin{array}{l}\text { Desarrolla su propuesta y la } \\
\text { presenta en formato video. }\end{array}$ & $\begin{array}{l}\text { Evaluación mediante rúbrica } \\
\text { de la video comunicación } \\
\text { (profesorado). }\end{array}$ \\
\hline $\begin{array}{l}\text { Participar de manera } \\
\text { apropiada en foros de } \\
\text { discusión científicos. }\end{array}$ & $\begin{array}{l}\text { Interviene en cada uno de los foros } \\
\text { de discusión. }\end{array}$ & $\begin{array}{l}\text { Evaluación mediante rúbrica } \\
\text { del grado de participación en } \\
\text { el foro y capacidad crítica } \\
\text { (profesorado). }\end{array}$ \\
\hline
\end{tabular}


El plan de acción, cronograma y espacios virtuales para el trabajo colaborativo propuesto desde el inicio de la actividad de innovación docente queda resumido en la Fig. 1. Se trata de minimizar la intervención del profesor y concretarla en proporcionar diferentes pautas y herramientas propias de la elaboración de una comunicación para un congreso on-line. De este modo delega en el estudiante, quien trabaja en dos direcciones: de manera individual, comunicándose con el profesor a través de la tutoría presencial y electrónica; y de manera colaborativa, por interacción con el resto de los compañeros. El estudiante se sitúa al nivel de un revisor que debe evaluar y sugerir correcciones de dos resúmenes de sendos compañeros, y proporcionar un feedback positivo en los foros una vez visualizadas las video comunicaciones subidas al espacio UMUbox de la Universidad de Murcia, de características similares al servicio de alojamiento Dropbox.

\begin{tabular}{|c|c|c|c|c|}
\hline & $24 / 10-06 / 11$ & | 07/11 - 13/11 & $14 / 11-12 / 12$ & $13 / 12-18 / 12$ \\
\hline 文 & Aula virtual (Tarea) & Aula virtual (Tarea) & UMUbox & Aula virtual (Foro) \\
\hline 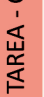 & $\begin{array}{l}\text { Envío de resumen y } \\
\text { bibliografía básica. }\end{array}$ & $\begin{array}{l}\text { Envío de la evaluación } \\
\text { de dos resúmenes. }\end{array}$ & $\begin{array}{l}\text { Envío de video } \\
\text { comunicaciones. }\end{array}$ & $\begin{array}{l}\text { Intervención en foros } \\
\text { de discusión. }\end{array}$ \\
\hline 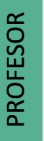 & $\begin{array}{l}\text { Presentación de las } \\
\text { bases de datos. } \\
\text { Pautas para elaboración } \\
\text { del resumen. }\end{array}$ & $\begin{array}{l}\text { Retroalimentación } \\
\text { resúmenes. } \\
\text { Criterios para } \\
\text { evaluación por pares. }\end{array}$ & $\begin{array}{l}\text { Tutorías electrónicas } \\
\text { individuales. }\end{array}$ & $\begin{array}{l}\text { Apertura y moderación } \\
\text { de foros de discusión. }\end{array}$ \\
\hline 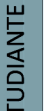 & $\begin{array}{l}\text { Revisión bibliográfica. } \\
\text { Elaboración de } \\
\text { resumen. }\end{array}$ & $\begin{array}{l}\text { Revisión por pares de } \\
\text { dos resúmenes de } \\
\text { sendos compañeros. }\end{array}$ & $\begin{array}{l}\text { Planificación, grabación } \\
\text { y edición de video } \\
\text { comunicación. }\end{array}$ & $\begin{array}{l}\text { Feedback a compañeros } \\
\text { en foros de discusión. }\end{array}$ \\
\hline
\end{tabular}

Fig. 1 Plan de acción y cronograma del proyecto de innovación.

Para la evaluación y evolución de nuestro proyecto de innovación elaboramos tres encuestas:

En la encuesta inicial, que tiene lugar en la clase presencial de presentación del proyecto, valoramos tres aspectos: el grado de conocimiento del estudiante acerca de las bases de datos para la investigación en Bellas Artes, su experiencia en la redacción y presentación de propuestas de investigación a congresos y/o revistas especializadas, y su participación en comités científicos.

Tras finalizar la segunda tarea abrimos dos foros de discusión en el Aula Virtual con sendas preguntas acerca del desarrollo de la parte teórica de la asignatura. La finalidad es que el estudiante adquiera las habilidades de expresión escrita y comunicación oral en público, al mismo tiempo que ensaya la mecánica de participación en un congreso on-line. 
La encuesta final, propuesta una vez concluida la asignatura y cumplimentada a través del Aula Virtual como autoevaluación, consta de nueve preguntas sobre la apreciación del grado de adquisición de las competencias planteadas en la asignatura a través del ABP $(5=$ máximo grado de adquisición de habilidades). Una última cuestión de respuesta libre bajo el enunciado "Indica cualquier aportación o comentario que desees transmitir con el fin de mejorar los resultados de adquisición de habilidades en la asignatura" nos permite evaluar el proyecto innovación y el aprovechamiento de las actividades propuestas para la asignatura.

\section{Resultados}

Los resultados tangibles del proyecto de innovación basado en la metodología ABP quedan reflejados en las encuestas inicial y de autoevaluación final, si bien las evaluaciones intermedias son tenidas en cuenta como referencia de la evolución del proyecto y como feedback hacia el estudiante.

La encuesta inicial, realizada a los doce alumnos matriculados en el curso 2018/2019, se resume en la Fig. 2.

Apreciamos que las fuentes bibliográficas más utilizadas para elaborar sus trabajos de investigación son libros especializados y páginas web, y que las menos utilizadas son actas de congresos (Fig. 2.a).

En la discusión conjunta sobre los resultados de esta encuesta, los alumnos manifiestan que los libros les son útiles a la hora de entrar en materia de la temática objeto de investigación y que las páginas web son una forma cómoda y rápida de consulta, aun siendo conscientes de que la publicación en internet no está sujeta a los filtros propios de la literatura científica (Fig. 2.b). Solamente cuatro de los doce estudiantes consultaron, previamente a su participación en este proyecto, los catálogos Alba y Xabio. Estos catálogos son, respectivamente, fuentes de datos bibliográficos de calidad de la Universidad de Murcia y documentos digitalizados de la comunidad científica. El desconocimiento de la disponibilidad de estas fuentes es uno de los motivos por los que siete de los doce estudiantes no habían accedido a textos procedentes de actas de congresos, los cuales están catalogados y disponibles en las bases de datos de centros de investigación.

Por último, su experiencia en la difusión de sus investigaciones en contextos científicos, salvo la de un estudiante de trayectoria investigadora consolidada, es escasa (Fig. 2.c). Este hecho se corresponde con estudiantes que se inician en postgrado y que se encuentran aún en fase de información acerca del área temática que desarrollarán en su futura Tesis Doctoral. 
Aprendizaje Basado en Proyectos (ABP). Congreso on-line como metodología de investigación integral aplicada a estudiantes de postgrado en Bellas Artes.

\section{2.a. Fuentes bibliográficas}

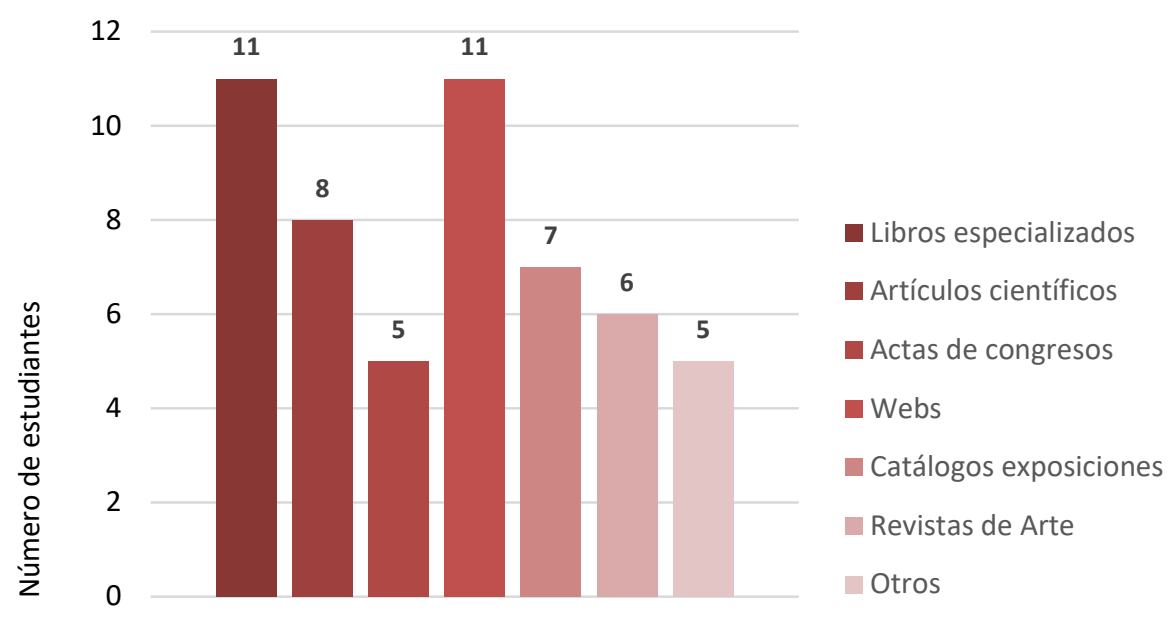

\section{2.b. Catálogos bibliográficos}

12

10

8

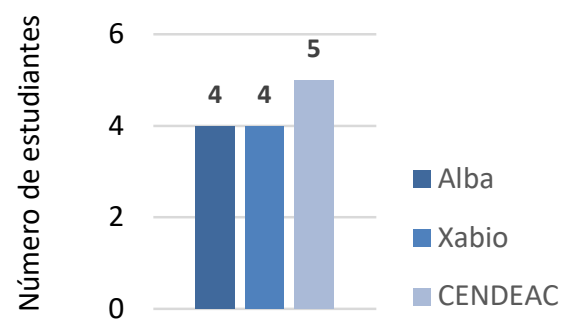

\section{2.c. Difusión científica}

12

10

8

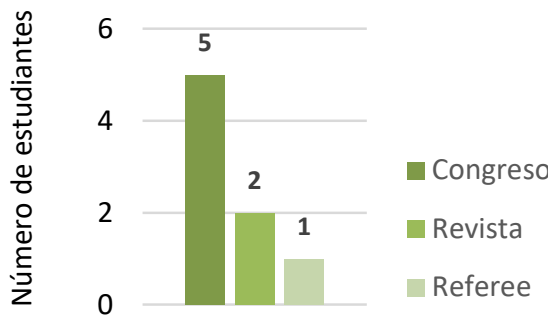

Fig. 2 Resultados de la encuesta inicial realizada a los doce alumnos matriculados en la asignatura Metodología e Investigación del Máster Universitario en Producción y Gestión Artística, 2018/2019.

En las nueve preguntas de la evaluación final enfocadas a conocer el grado de adquisición de competencias obtuvimos medias superiores a 4 sobre 5 (Fig. 3). Respecto a la última pregunta, en la que el alumno responde de manera abierta, cabe destacar, además de la consideración de un acierto el ABP vertebrado en un congreso on-line, la necesidad de asignar más créditos a esta asignatura obligatoria, prolongándola a lo largo de todo el curso académico, es decir, dos cuatrimestres en lugar de uno. De esta manera, los estudiantes dispondrían de un espacio virtual en el que basar la investigación de cada uno de los 
proyectos solicitados en el resto de las asignaturas del Máster, tanto las pertenecientes al primer cuatrimestre como las del segundo.

\section{Autoevaluación sobre el grado de adquisición de competencias por parte del estudiante}

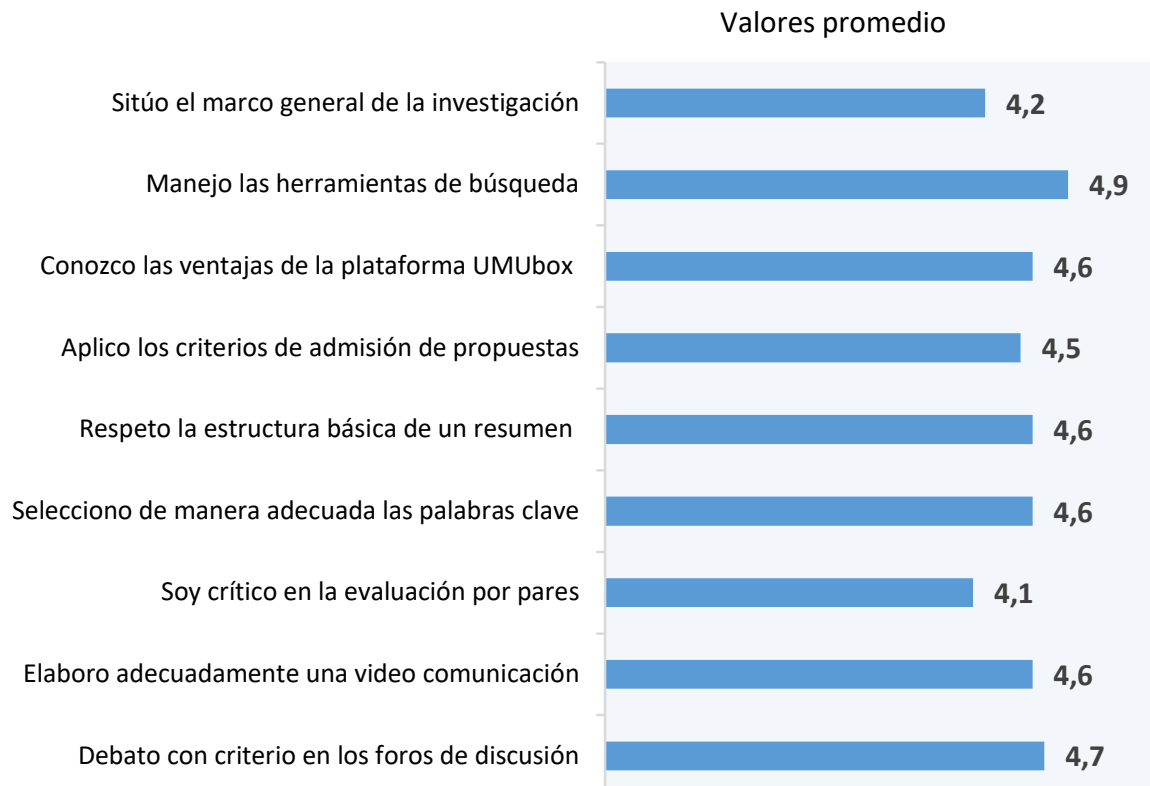

Fig. 3 Resultados de la encuesta final de autoevaluación realizada a los doce alumnos matriculados en la asignatura Metodología e Investigación del Máster Universitario en Producción y Gestión Artística, 2018/2019.

Los resultados acerca del grado de satisfacción del proyecto de congreso on-line coinciden con los obtenidos por el Grupo de Investigación "Comunicación y sociedad digital" del Plan Propio de la Universidad Internacional de la Rioja (Berlanga Fernández y Merino Arribas, 2014). Este grupo plantea también un congreso on-line en el que se incluyen, además, conferencias de invitados expertos. Los estudiantes de este estudio deben elaborar una ponencia bajo el esquema generalizado IMRYD (Introducción, Objetivos, Hipótesis, Métodos de investigación, Resultados, Discusión, Conclusiones y Bibliografía) dentro de unos ejes temáticos concretos. Sin bien no exponen su ponencia ni participan en la corrección por pares, actúan como moderadores en mesas redondas organizadas por líneas siguiendo un modelo $100 \%$ on-line.

\section{Conclusiones}

Los estudios superiores en Bellas Artes constituyen un contexto idóneo en el que adaptar una metodología ABP vertebrada en torno a un congreso on-line dadas, por un lado, las 
características y el nivel del estudiante de postgrado, y por otro, las posibilidades que ofrece el trabajo a través de las Tecnologías de la Información y la Comunicación (TIC).

Respecto al primer aspecto, es obvio que la mayor carga de trabajo recae en el estudiante, que debe conectar diferentes saberes para presentar un producto artístico tangible. Tras cuatro años de estudios de Grado en los que ha adquirido unas competencias y habilidades propias de las Bellas Artes, se encuentra en el momento idóneo para desarrollar su capacidad de investigación teórica. Esta capacidad constituye, además, una iniciación para sus estudios de doctorado, pues a lo largo del Grado las asignaturas se trabajan de manera independiente, por medio de proyectos a corto plazo y encuadrados generalmente en una de las tres áreas de Dibujo, Escultura y Pintura.

En lo referente al manejo de las TIC, los estudiantes de postgrado de Bellas Artes están habituados a utilizar las tecnologías como modo de comunicación y también en la producción de proyectos de componente digital. Por tanto, el manejo de plataformas y de redes de comunicación no solo no suponen un problema, sino que son un punto de encuentro para el trabajo colaborativo, una de las principales características de la metodología ABP como emulación de la realidad social.

\section{Referencias}

BERLANGA FERNÁNDEZ, I. y MERINO ARRIBAS, A. (2014). "Investigación 2.0. Un modelo de congreso en la sociedad post-digital" en Historia y Comunicación Social, vol. 19, pp. 15-26.

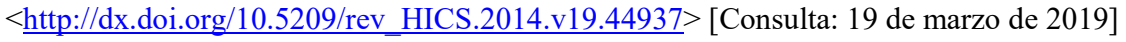

JONES, N. F., RASSMUSSEN, C. M. Y MOFFITT, M. C. (1997) Real-life problem solving: A collaborative approach to interdisciplinary learning. Washington: American Psychological Association

TORREGO EGIDO, L. Y MÉNDEZ ROMERO R.A. (coord.) (2018). "Un acercamiento al aprendizaje basado en proyectos, cien años después de "The project method", de W.H. Kilpatrick" en Revista Electrónica Interuniversiaria de Formación del Profesorado, (REIFOP), vol. 60, pp. 1-178.

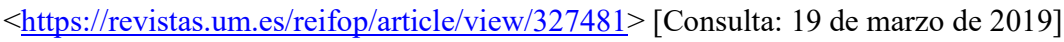

